\title{
Factors influencing the efficiency of a marker-assisted introgression programme in Merino sheep
}

\author{
Sonja DOMINIK ${ }^{\mathrm{a}, \mathrm{c} *}$, John HENSHALL $^{\mathrm{a}, \mathrm{c}}$, Julian O'GRADY ${ }^{\mathrm{a}, \mathrm{c}}$, \\ Karen MARSHALL ${ }^{\text {b,c }}$ \\ ${ }^{\text {a }}$ CSIRO Livestock Industries, Armidale, Australia \\ ${ }^{\mathrm{b}}$ Department of Animal Science, University of New England, Armidale, Australia \\ ${ }^{\mathrm{c}}$ Australian Sheep Industry CRC, Armidale, Australia
}

(Received 1st September 2006; accepted 12 March 2007)

\begin{abstract}
This study investigated a marker-assisted introgression programme in Australian Merino sheep. The goal was to introgress an allele with a large negative effect on fibre diameter into a Merino flock possessing medium average fibre diameter. The influence of two factors was explored: the strategy used to select animals from the purebred and backcross line for backcrossing purposes and the use of selection on background markers to accelerate the return to the purebred line's genome. The results were compared to introgression based on EBVs only. Introgression using EBVs only produced almost the same response in the dollar index as marker-based introgression methods. However, this study did not account for some of the costs associated with implementing the programmes, including the costs of phenotyping and genotyping. Given that the cost of measuring fibre diameter is low, it was concluded that introgression on EBVs only would be the preferred method since the marginal profit of markerassisted introgression would not be large enough to cover the additional cost of genotyping. In marker-assisted introgression, reciprocal crossing of male and female selection candidates from the backcross and the purebred line was the most advantageous strategy from a practical and profit point of view. Selection for background markers was less profitable in this study than recovering the donor genome by selection on phenotype.
\end{abstract}

marker-assisted introgression / Merino sheep / sheep breeding

\section{INTRODUCTION}

The process of introgression in the field of animal breeding describes the transfer of a favourable allele of a gene from a donor line of animals into a purebred line that does not carry this allele. The first step of an introgression

*Corresponding author: Sonja.dominik@csiro.au 
programme is to cross a purebred line with a donor line. In the following generations, the crossbred animals that are heterozygous for the favourable allele are backcrossed to the purebred line to increase the proportion of the genome of the purebred line. Marker-assisted introgression (MAI) is applied with the same intent. However, through the use of molecular markers that are linked to the favourable allele, carriers can be identified and the accuracy of selection of the appropriate breeding animals is increased compared to phenotypic selection.

To date, marker-assisted introgression has not been widely commercially applied in the sheep industry. Genetic markers are available for major genes that influence fertility and carcass characteristics in sheep (e.g. the Booroola gene [13] and Callipyge gene [5]), but because of their extreme phenotypic expression, they are not widely applied in breeding programmes. In general, there is a paucity of highly accurate genetic markers linked to genes that influence economically important characteristics in sheep. Currently, there is only a small number of published QTL for fibre diameter and other wool production and quality characteristics $[1,2,8-10,12]$.

Factors influencing the efficiency of MAI programmes in livestock have been discussed by a number of authors. In the case of a situation concerning pig breeding, Visscher and Haley [15] concluded that a genetic lag between the purebred line and the introgression line occurs during the backcrossing process. As method to reduce the genetic lag, they proposed selection on markers that cover the purebred line's genome. This strategy was found to accelerate the recovery of the purebred line's background genes and reduce the required number of years of backcrossing.

A study by van der Waaij and van Arendonk [14] focused on an MAI programme in cattle. In agreement with Visscher and Haley [15], they found that the number of backcross generations impacts on the genetic difference between the introgressed and purebred line. Visscher et al. [16] further concluded that the use of reproductive technologies has an effect on the number of backcross generations, as does the initial difference in trait expression between the donor and recipient line. They concluded that the maximum genetic advantage of the introgressed line differs from the maximum economic advantage [16]. The degree of divergence is likely to differ between species due to variation in constraints (such as fecundity) and to variation in economic factors. This highlights the importance of assessing introgression programmes in speciesspecific breeding programmes that include the relevant economic background in the evaluation. 
MAI could provide a useful breeding strategy for the Australian Merino industry. The production aims of the Australian Merino industry are geographically differentiated due to differences in production environments and to industry beliefs regarding sheep survival, wool production and quality. In recent years, research has shown that moving to fine wool production in areas, which have previously produced medium wool has positive effects on wool enterprise returns with no major adverse effects on wool quality [7]. This is due to the higher average premium paid for fine wool. If Merino producers in traditional medium to strong wool production areas decide to produce sheep with finer wool, MAI could be considered as a potential breeding tool to reduce the mean average diameter without compromising clean fleece production, if genetic markers were available.

The aim of this study was to explore factors influencing genetic and economic gain in MAI programmes using stochastic computer simulation based on a realistic Australian wool sheep industry scenario. Two aspects concerning the backcross generation were investigated: first, the effect of different backcrossing strategies on the economic gain in MAI programmes and second, the effect of selection on background markers to recover the purebred line's genome as quickly as possible. The results were compared with introgression based on EBVs only.

\section{MATERIALS AND METHODS}

\subsection{Simulation study}

\subsubsection{General background}

The scenario that serves as background for this study assumed that an allele with a large negative effect on fibre diameter is introduced from a fine wool Merino donor line into a medium wool Merino purebred line. This allele can be identified through genetic markers that are closely linked. Different breeding and selection strategies were individually evaluated for their results in the last year (year 20) of an introgression programme. The comparison of the results was based on the difference in profit between the introgression product and the initial purebred line due to the difference in performance in fibre diameter (FD) and fleece weight (CFW). Technological costs for genotyping and advanced reproduction were not accounted for.

Fifty repetitions of the computer simulation were run for each of the breeding strategies. Each repetition comprised 20 years of flock breeding. In our study, the allele effect size of the QTL equals one micron and the QTL does 


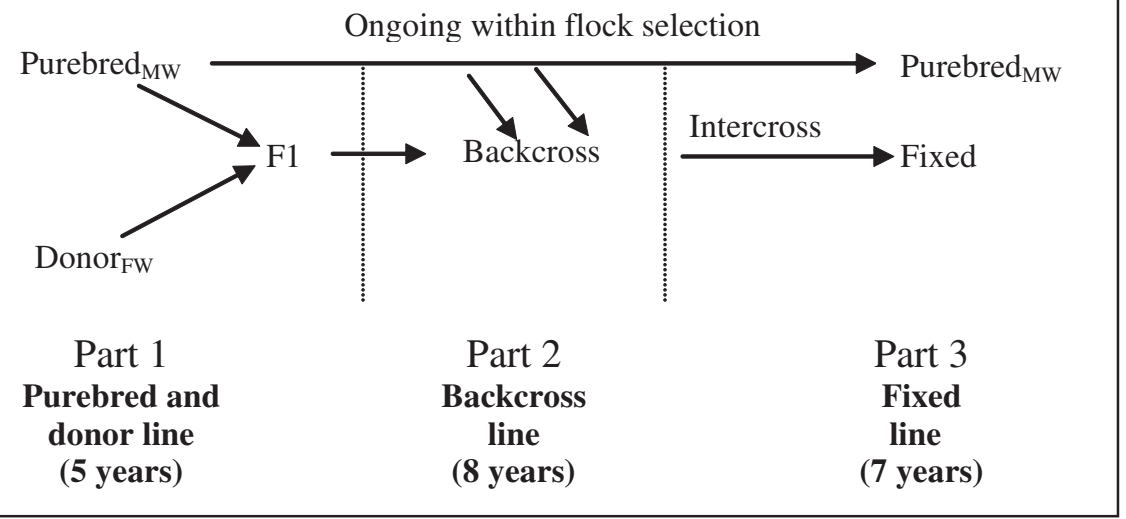

Figure 1. Breeding structure for the simulation study - introgression by using a donor/fine wool line (donor ${ }_{\mathrm{FW}}$ ) and a purebred recipient line/medium wool line ( purebred $_{\mathrm{MW}}$ ).

not have any effect on other traits. The QTL effect with $67.7 \%$ of a phenotypic standard deviation was large in comparison to published effect sizes $[1,8,10]$. It was assumed that no recombination occurred between the marker and the QTL.

Breeding values were estimated with ASREML [6] applying an animal model that fitted birth year and line (e.g. purebred, backcross) as fixed effects. Full pedigree information was used.

\subsubsection{Breeding structure}

The initial generations in the simulation were aimed at the production of a flock with overlapping generations, a range of age classes and realistic within flock variance structures. Each of the initial two lines (fine and medium wool) started with a population of 500 animals and was randomly bred for 10 years. Each year, five rams were randomly selected and mated to all available ewes. The rams were culled after two years and ewes after five years. This process provided a starting population of around 1250 animals.

Figure 1 schematically outlines the general breeding structure that was applied in this study, broken down into three parts. The numbers of male and female selection candidates in the different parts and lines of the breeding structure are summarised in Table I.

In Part 1, the breeding structure comprised two lines of sheep: a fine wool Merino donor line, which is homozygous for the favourable allele with 
Table I. Number of males and females selected each year in the purebred ${ }_{\mathrm{MW}}$, backcross and fixed line in the breeding structure of the introgression program.

\begin{tabular}{|c|c|c|c|c|c|}
\hline Line & Sire line & Dam line & $\begin{array}{l}\text { Backcross } \\
\text { strategy }\end{array}$ & $\begin{array}{l}\mathrm{Nb} \text { of } \\
\text { males }\end{array}$ & $\begin{array}{l}\mathrm{Nb} \text { of } \\
\text { females }\end{array}$ \\
\hline Purebred $_{\mathrm{MW}}$ & Purebred $_{\mathrm{MW}}$ & Purebred $_{\mathrm{MW}}$ & & 5 & 250 \\
\hline F1 & Donor $_{\mathrm{FW}}$ & Purebred $_{\mathrm{MW}}$ & & 1 & 55 \\
\hline \multirow[t]{4}{*}{ Backcross } & Backcross & Purebred $_{\mathrm{MW}}$ & Strategy 1 & 1 & 70 \\
\hline & Purebred $_{\mathrm{MW}}$ & Backcross & (reciprocal) & 1 & 50 \\
\hline & Backcross & Purebred $_{\mathrm{MW}}$ & Strategy 2 & 1 & 120 \\
\hline & Purebred $_{\mathrm{MW}}$ & Backcross & Strategy 3 & 1 & 120 \\
\hline Intercross & Backcross & Backcross & & 1 & 55 \\
\hline Fixed & Fixed & Fixed & & $2^{a}$ & $50^{\mathrm{a}}$ \\
\hline
\end{tabular}

${ }^{a}$ These numbers increase with increasing number of selection candidates.

a negative effect on fibre diameter, and a medium wool Merino purebred line $\left(\right.$ purebred ${ }_{\mathrm{MW}}$ ), which is homozygous for the unfavourable allele. In the purebred $_{\mathrm{MW}}$ line, the breeding programme was based on within flock selection on EBVs for fleece weight and fibre diameter. Rams and ewes were culled at four and seven years of age, which resulted in a larger proportion of females. Each year, five rams and 250 ewes were selected. In the next step, a first cross (F1 generation) between the two lines was produced, which was $100 \%$ heterozygous for the favourable allele.

In Part 2, the breeding structure contrasts the purebred $\mathrm{MW}_{\mathrm{W}}$ line with the "backcross line". The establishment of the backcross line involved eight years of backcrossing animals to the purebred ${ }_{\mathrm{MW}}$ line. This process aimed at recovering the purebred line's genome.

In Part 3, a so-called "fixed line" was produced. The fixed line is the outcome of an intercross between backcross animals that results in a proportion of progeny that are homozygous for the favourable allele. These enter the fixed line, and the success of the programme can be evaluated by comparing these to the purebred ${ }_{\mathrm{MW}}$ line. Selection on production traits was conducted by using the same index in all lines (details are described in Sect. 2.4).

\subsubsection{Genetic and phenotypic parameters}

The stochastic computer simulation was based on literature estimates $[3,11]$ of genetic and phenotypic parameters for fibre diameter (FD) and clean fleece

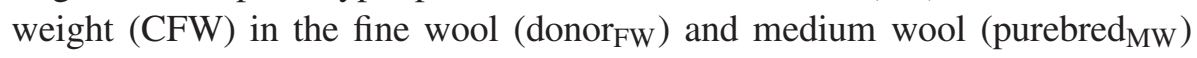


Table II. Means, phenotypic and genetic variances $\left(\mathrm{V}_{\mathrm{p}}\right.$ and $\left.\mathrm{V}_{\mathrm{a}}\right)$ for fine and medium wool Merino sheep for clean fleece weight (CFW) and fibre diameter (FD) $[3,10]$.

\begin{tabular}{lllll}
\hline & \multicolumn{2}{l}{ Fine wool Merino sheep } & \multicolumn{2}{l}{ Medium wool Merino sheep } \\
\hline & CFW & FD & CFW & FD \\
\hline Mean & $2.5 \mathrm{~kg}$ & $17 \mu \mathrm{m}$ & $4 \mathrm{~kg}$ & $22 \mu \mathrm{m}$ \\
$\mathrm{V}_{\mathrm{a}}$ & 0.02 & 0.77 & 0.16 & 1.55 \\
$\mathrm{~V}_{\mathrm{p}}$ & 0.06 & 1.13 & 0.37 & 2.18 \\
\hline
\end{tabular}

Table III. Genetic and phenotypic parameters for fine and medium wool Merino sheep for clean fleece weight (CFW) and fibre diameter (FD) - heritabilities in bold, phenotype correlations $\left(r_{p}\right)$ above the diagonal and genetic correlations $\left(r_{g}\right)$ below the diagonal $[3,11]$.

\begin{tabular}{clccc}
\hline & \multicolumn{2}{l}{ Fine wool Merino sheep } & \multicolumn{2}{l}{ Medium wool Merino sheep } \\
\hline & CFW & FD & CFW & FD \\
\hline CFW & $\mathbf{0 . 2 9}^{*}$ & $0.20^{*}$ & $\mathbf{0 . 4 2} \pm \mathbf{0 . 0 2}$ & $0.25 \pm 0.03$ \\
FD & $0.14^{*}$ & $\mathbf{0 . 6 8}^{*}$ & $0.31 \pm 0.03$ & $\mathbf{0 . 7 1} \pm \mathbf{0 . 0 2}$ \\
\hline
\end{tabular}

* No standard errors available.

Merino flocks. The estimates and industry representative means and variances are summarised in Tables II and III.

Genetic and phenotypic variances for CFW and FD differ between medium and fine wool sheep populations. A polygenic component of the genetic variances for all lines was simulated using variance component estimates from fine wool sheep. The higher means and genetic variances in medium wool sheep were assumed to be due to the action of a finite number of genes, each with two alleles and with additive gene action (i.e. no dominance effect was simulated).

For each gene, base animals in the fine wool population were simulated as homozygous for the allele of negative effect, so these genes did not contribute to the genetic variance in that line. In the base animals for the medium wool population, the alleles were simulated at a frequency of 0.5 , resulting in both higher means and higher genetic variances in this line. Two classes of allelic substitution effects are required to account for the correlation structures. The number of genes required and allele substitution effects were derived using formulae for allele effects [4]. These are summarised in Table IV. 
Table IV. Allelic substitution effect $(\alpha)$ on FD and CFW for a specified number of genes (n).

\begin{tabular}{lll}
\hline & $\alpha_{\text {class } 1}$ & $\alpha_{\text {class } 2}$ \\
\hline $\mathrm{n}$ & 9 & 9 \\
$\mathrm{CFW}$ & -0.00898 & 0.176096 \\
$\mathrm{FD}$ & 0.368099 & 0.192687 \\
\hline
\end{tabular}

The following equations describe the components of the phenotypic variances of the base animals of the fine and medium wool line:

$$
\begin{gathered}
\mathrm{V}_{\mathrm{p}}=\mathrm{V}_{\mathrm{aFW}}+\mathrm{V}_{\text {Genes }}+\mathrm{V}_{\mathrm{e}} \\
\mathrm{V}_{\text {Genes }}=\sum_{\mathrm{i}=1}^{\mathrm{n} 1} 2 \mathrm{pq} \alpha_{1}^{2}+\sum_{\mathrm{i}=1}^{\mathrm{n} 2} 2 \mathrm{pq} \alpha_{2}^{2},
\end{gathered}
$$

where $\mathrm{V}_{\mathrm{p}}$ is the phenotypic variances for the fine or medium wool line and $\mathrm{V}_{\mathrm{aFW}}$ is the gene variance of the fine wool line (Tab. II), $\mathrm{V}_{\mathrm{Genes}}$ is the variance due to allele substitution effects $\left(\alpha_{1}\right.$ and $\left.\alpha_{2}\right)$ of a specified number of genes $(\mathrm{n} 1$ and $\mathrm{n} 2)(\mathrm{Tab} . \mathrm{IV})$ at frequency $\mathrm{p}(\mathrm{p}=0$ for fine wool, $\mathrm{p}=0.5$ for medium wool and $q=1-p), V_{e}$ is the error variance for the fine or medium wool line (Tab. II).

The error variances for crossbred animals were assigned using a linear interpolation, with the variance dependent on the proportion of medium wool ancestry, as outlined below:

$$
\mathrm{V}_{\mathrm{eBC}}=\mathrm{V}_{\mathrm{eFW}}+\pi \times\left(\mathrm{V}_{\mathrm{eMW}}-\mathrm{V}_{\mathrm{eFW}}\right),
$$

where $\mathrm{V}_{\mathrm{eBC}}$ is the error variance in the backcross line, $\mathrm{V}_{\mathrm{eFW}}$ and $\mathrm{V}_{\mathrm{eMW}}$ are the error variances for the fine wool and medium wool line (Tab. II), $\pi$ is the proportion of medium wool ancestry.

\subsubsection{Economic parameters}

Historical data on the sale price of wool from 1992 to 2003 were used to estimate revenue as a function of fleece weight and fibre diameter. Expenses were assumed to increase linearly with decreasing fleece weight. Under the assumption that the index used in industry (80\% emphasis on fleece weight, $20 \%$ on fibre diameter) is optimal, an equation for profit in dollars per sheep as a function of fibre diameter was derived:

$$
\text { Profit }=\text { CFW } \times(2123.446-90 \times \text { FD }) .
$$


Although the profit function is non-linear and the variance components differ between the fine and medium wool lines and are not constant in the backcross line, a constant 80:20 ratio for selection emphasis on fleece weight and fibre diameter was used to select parents in the simulation studies.

\subsection{Breeding strategies}

Within the general breeding structure described in Section 2.1.1., different crossing strategies were applied in the backcross line (Part 2, Fig. 1). Figure 2 summarises the different strategies.

Three different backcross strategies were considered where males and females are sourced from either of the lines. In general, selection of animals from purebred $\mathrm{d}_{\mathrm{MW}}$ for within-flock breeding takes priority over selection for backcrossing. Strategy 1 was a reciprocal cross between males and females from the purebred line with males and females from the backcross line. In Strategy 2 all males were selected from the backcross line and all females from the purebred $\mathrm{MW}_{\mathrm{MW}}$ line. Selection of females from the purebred $_{\mathrm{MW}}$ was restricted to the genetically second best animals because the best were used for flock replacement of the purebred $d_{M W}$ line. Strategy 3 reversed the concept and males were selected from the purebred line and mated to backcross line females. The genetically best males from purebred $\mathrm{MW}_{\mathrm{MW}}$ can be used through artificial insemination. However, Strategy 3 can only be applied in combination with multiple ovulation embryo transfer (MOET) because of the low number of females with the appropriate genotype available for selection.

\subsection{Methods of selection}

\subsubsection{Selection on BLUP breeding values}

Selection on BLUP breeding values is a means of introgressing the favourable allele without the use of genetic markers, by assuming that the breeding values account for the QTL effect. The aim is to investigate the efficiency with which selection on EBVs alone increases the frequency of the favourable allele. The scenario where selection is based on EBVs alone is subsequently referred to as "BLUPonly".

\subsubsection{Marker assisted introgression}

In making selection decisions in MAI, phenotype and pedigree data are augmented by the information on the genotype that an animal carries at marker loci 


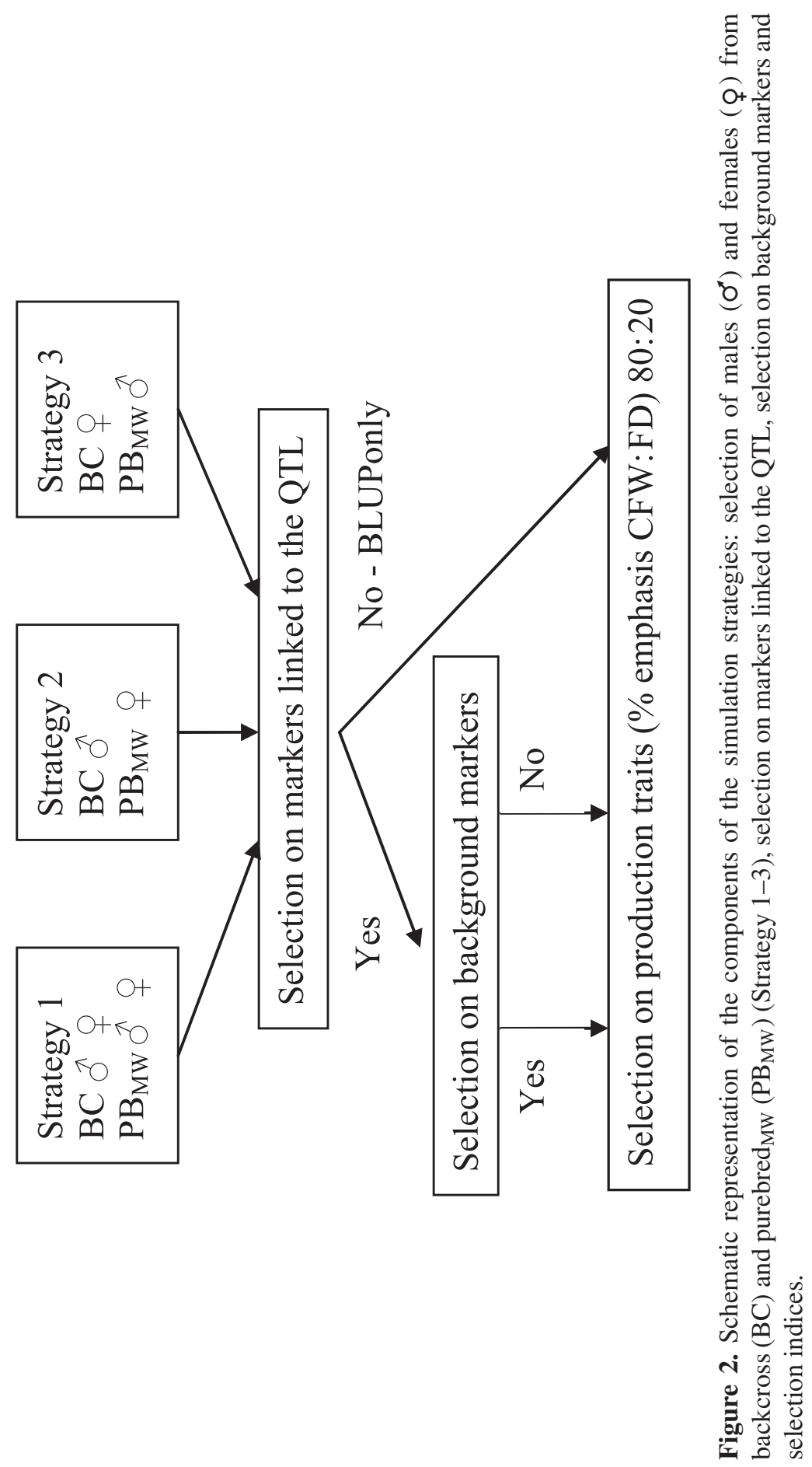


that are linked to the QTL. The MAI scenario in this study describes a special case of marker and QTL relationship, where there is no recombination between the marker and the QTL. Selection candidates that are heterozygous for the favourable genotype at the marker loci are selected as parents of the next generation. Therefore truncation selection was the first selection step based on the zygosity status for the QTL, followed by selection decisions based on performance in the 80:20 trait index.

\subsubsection{Marker assisted introgression plus background marker selection}

Selection on markers that are linked to genes from the medium wool line can have a favourable effect on the speed with which medium wool background genes accumulate and can reduce the number of required backcrosses [15]. In order to simulate the effect of background marker selection it is necessary to make some assumptions regarding the distribution of genes affecting the traits of interest over the genome. For this study, the following simple approach was taken i.e. simulating 18 line specific markers at recombination rates of 5\% from the 18 finite locus genes described in Section 2.1.3. Each of the strategies of backcrossing was simulated with and without selection on background markers. In the selection process, first truncation selection was performed based on the zygosity status for the QTL, with only heterozygous animals being selected. For the background marker, the second selection step was to select the animals with the largest proportion of markers of medium wool ancestry. Trait index performance, which is based on phenotype and pedigree, was only considered when animals were equal for a proportion of background markers.

\section{RESULTS}

\subsection{Base scenario - Purebred medium wool line}

The purebred medium wool line serves as a base line comparison for the outcome of the MAI process in the fixed line. Figure 3 shows the progress in FD, CFW and profit over the 15 years, starting in year 6 of the breeding programme. Year 6 of the breeding programme is the first dot on the right. At the beginning of the breeding programme (result not shown), the purebred $\mathrm{MW}_{\mathrm{W}}$ line had wool with a FD of $22 \mu \mathrm{m}$ and $4 \mathrm{~kg}$ CFW, which returned $\$ 5.72$ per sheep. Selection caused an almost linear increase in CFW and profit and a linear decrease in FD. By year 14 of the breeding programme, which is the first year in which a comparison with the fixed line becomes possible, CFW 


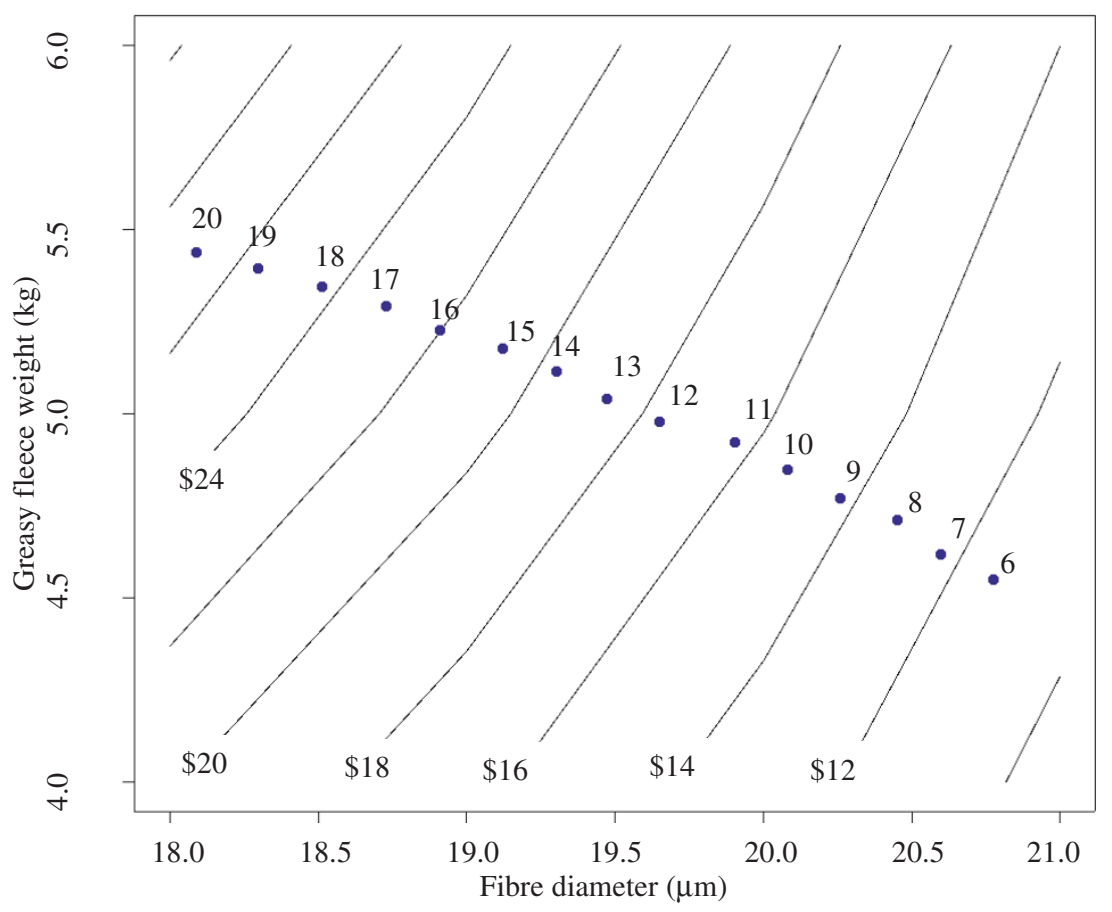

Figure 3. Progress of within flock selection in the purebred $_{\mathrm{MW}}$ line in FD and CFW over 15 years, starting at the production of the F1 cross (year 6), plotted across curves of the same profit (iso-economic curves).

had increased to $5.14 \mathrm{~kg}$ and FD reduced to $19.3 \mu \mathrm{m}$. These changes increased profit by $\$ 13.87$ per sheep compared to the first year. In the last year of the purebred breeding programme CFW reached $5.48 \mathrm{~kg}$, FD $18.11 \mu \mathrm{m}$ and profit increased by a further $\$ 7.43$ per sheep to $\$ 27.02$.

\subsection{Comparison of MAI to BLUPonly}

Selection in the backcross generation based on EBVs alone was tested for its impact on profit. All MAI breeding strategies of backcrossing were explored without background marker selection. Figure 4 shows the results from "BLUPonly" across backcross Strategy 1 to 3 in comparison to the backcross strategies evaluated with MAI. The results are presented as difference in profit in dollar between the purebred medium wool line and the evaluated introgression strategy. Overall BLUPonly performed worse than the MAI scenarios. 


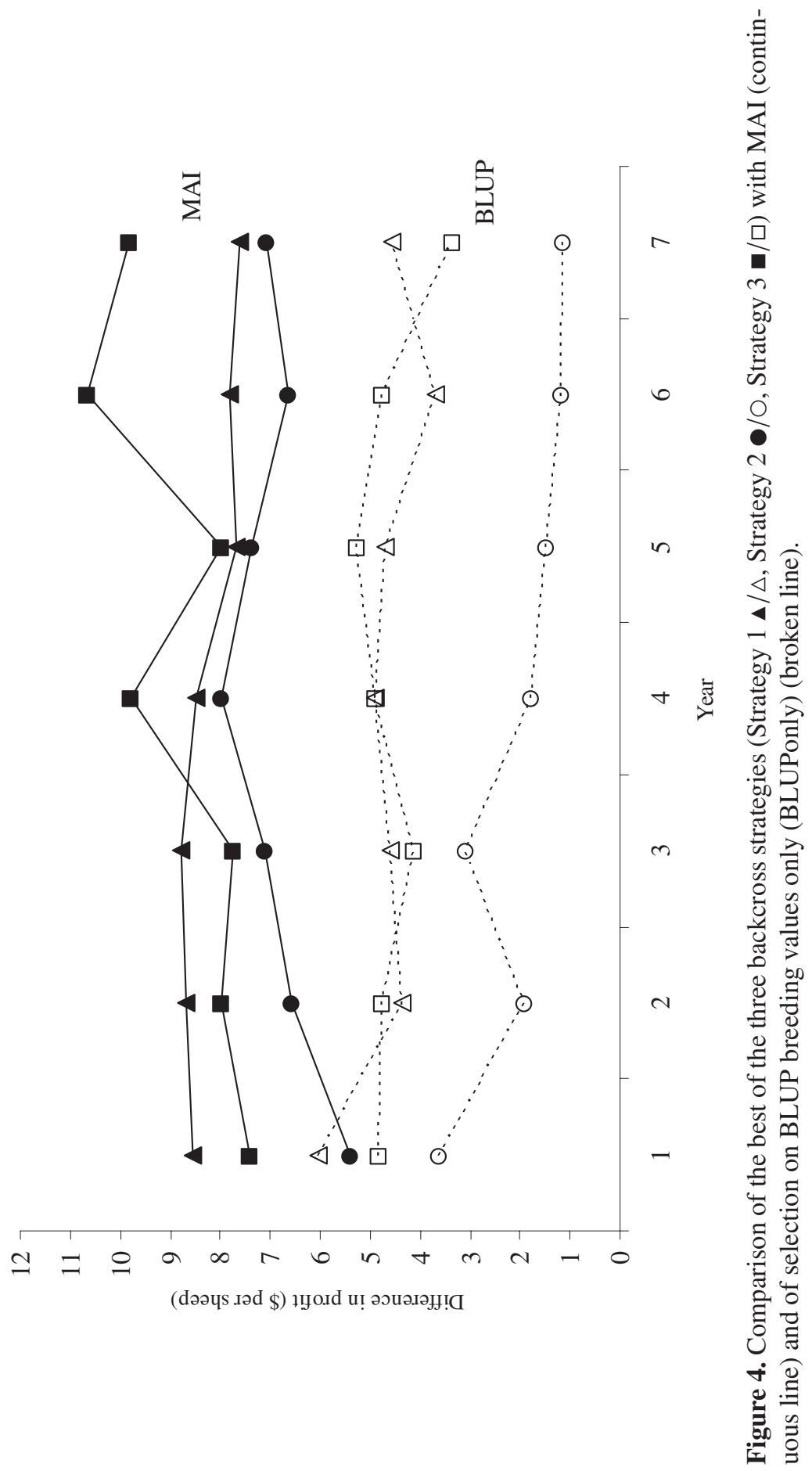


The best BLUPonly scenario yielded $\$ 2.50$ less than the least profitable MAI scenario in the final year of the breeding programme. The best MAI strategy was $\$ 5.26$ better than the best BLUPonly, however that result was found for Strategy 3, which would involve MOET. For the second best MAI, which was Strategy 1, the difference with BLUPonly was $\$ 3.02$. The most profitable BLUPonly scenario, which was based on backcross Strategy 1, resulted in $\$ 4.57$ more profit per sheep than within flock selection in the purebred $_{M W}$ line. The least profitable scenario for BLUPonly with backcrossing Strategy 2 had only an advantage of $\$ 1.16$ over the purebred ${ }_{\mathrm{MW}}$ line. Backcross Strategies 1 and 3 performed similar in BLUPonly.

\subsection{Impact of different strategies in the backcross generation}

The impact of three different backcrossing strategies on the profitability of the MAI process was investigated. Strategy 1 selected males and females from both the medium wool line and the backcross line for reciprocal crosses, Strategy 2 sourced females from the medium wool line and males from the backcross line and Strategy 3 did the reverse. Figure 5 shows the difference between the medium purebred and fixed line across all the different backcross strategies.

All MAI scenarios yielded more profit than within flock selection in the purebred $_{\mathrm{MW}}$ line; therefore all profit curves have positive values. Across all backcrossing strategies, with or without background marker selection, Strategy 3 was on average the most profitable option followed by Strategy 1, with Strategy 2 being the least profitable. Strategy 3 consistently yielded more profit per sheep in the last year of the breeding programme than the other strategies and Strategy 1 was more profitable than Strategy 2.

\subsection{Background marker selection}

The scenarios with and without background marker selection are shown in Figure 5. In general, the inclusion of background markers has a negative effect on the profit per sheep compared to scenarios without background marker selection. On average, scenarios without background marker selection produced $\$ 5.62$ more profit than scenarios with background selection the last year of the breeding programme. Strategies 1 and 2 did not perform very differently with background marker selection. Furthermore, Figure 5 shows that the three strategies performed similarly in the beginning with background marker selection, whereas without, the effect of the backcrossing strategy caused a marked difference. 


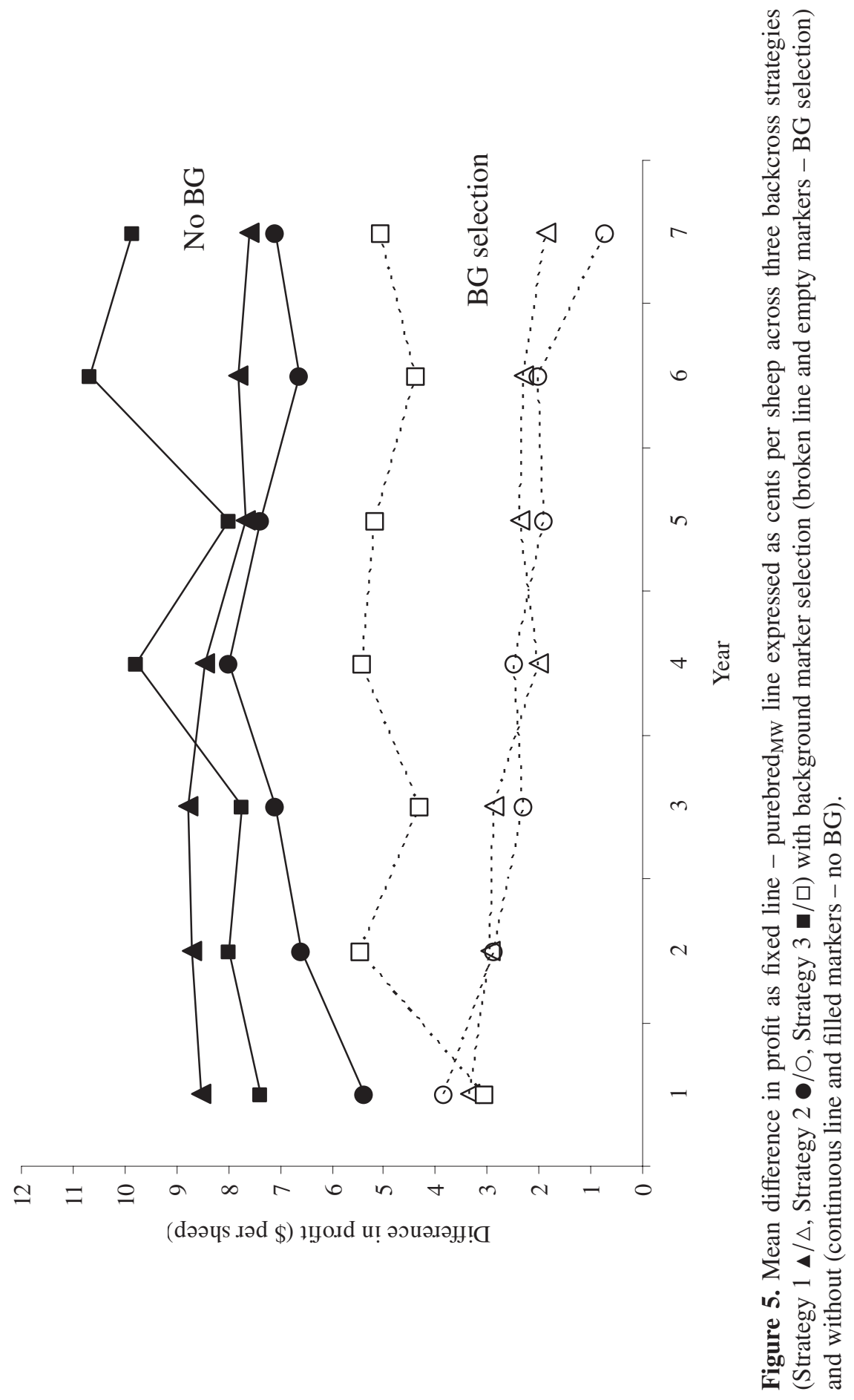




\section{DISCUSSION}

MAI will become an important breeding tool in the sheep industry when genetic marker technology reaches the commercial level. Currently, there are no studies available that examine factors affecting performance of MAI programmes in the case of a situation concerning sheep breeding. This study investigated two factors and their interactions that influence the backcross generation of an MAI breeding programme and therefore the overall success: first the selection strategy in the purebred and the backcross line and second the option of selecting on background markers covering the purebred line's genome. Finally, the results were compared to the genetic and economic progress of an introgression programme that bases selection decisions solely on estimated breeding values.

For the situation modelled in this study, selection on BLUP breeding values without using QTL information was competitive with a marker assisted introgression programme. Cost for genotyping and reproductive technologies were not taken into account in this study, but the margin gained through introgression based on markers and MOET was low compared to the cost that would have to be spent on the technologies involved. Overall, introgression based on the use of estimated breeding values yielded less profit than MAI, however, the difference between the approaches indicated a maximum allowable cost for genotyping and reproductive technologies. Current commercially available gene tests for cattle are approximately $\$ 85$ per animal and an estimate for single nucleotide polymorphism (SNP) or microsatellite marker genotyping is at around \$1 per marker. Compared to this, the margin in profit of MAI over BLUPonly of $\$ 3.02$ to $\$ 5.27$ is comparably small.

In this study, it was assumed that trait records for fibre diameter were available for all animals. Since the cost of measuring fibre diameter is small compared to the cost of genotyping, BLUPonly is probably the preferred strategy. This may be reversed for traits that are more expensive to measure.

The factor causing the most influence on the different backcrossing strategies in the MAI breeding programme was the availability of genetically superior animals for the backcrossing process. The availability of females for backcrossing from the purebred line was restricted because the use of females for production of replacement animals within the flock was given priority. The number of females in the backcross line was limited since only half were heterozygous for the QTL. Selection of males is unrestricted in either of the lines because the required numbers are low and artificial insemination allows the parallel use of sires for within flock breeding and backcrossing. The strategy that uses males from the purebred line through artificial insemination and 
females from the backcross line is the most profitable strategy, but practically expensive because advanced reproductive technologies are required. Therefore, a reciprocal cross of the backcross and the purebred line, would offer the highest overall return.

Several authors $[15,16]$ have investigated the effect of background marker selection to return to the purebred line's genome. In contrast with the conclusions in these studies, where background selection is advantageous, in our study, response was worse with background marker selection. There are a number of possible explanations for this. In our study, the QTL explained only a proportion of the differences between the lines for fibre diameter, with the remainder due to loci at which the favourable allele is at a higher frequency in the donor population. Background selection discriminates against these alleles, while BLUP EBV, as a secondary selection criterion after QTL genotypes, exploit this variance. This is particularly the case for class 1 alleles where there is no increase in fleece weight when selecting for the medium wool alleles but an increase in fibre diameter (Tab. IV). Consequently, selection on background markers was associated with unfavourable polygenic trait responses and the scenario as considered in this simulation study did not benefit from selection on background markers. It might be possible to improve this by reducing the emphasis on background selection, possibly estimating the appropriate weight from the observed regression of phenotype on proportion of medium wool alleles.

In conclusion, this study evaluated strategies of marker assisted introgression, and showed that selection on BLUP breeding values can be almost as effective for introgression of a favourable allele of a gene in a sheep breeding system. However, a well-implemented marker assisted introgression programme can provide greater control and accuracy of selection and may offer other logistical advantages to a breeding programme. It may also offer considerable advantages where the trait affected by the QTL is difficult or expensive to measure.

\section{ACKNOWLEDGEMENTS}

The work reported here was financially supported by the Australian Sheep Industry CRC.

\section{REFERENCES}

[1] Allain D., Schibler L., Mura L., Barillet F., Sechi T., Rupp R., Casu S., Cribiu E., Carta A., QTL detection with DNA markers for wool traits in a sheep backcross 
Sarda $\times$ Lacaune resource population, Proc. 8th World Congr. Genet. Appl. Livest. Prod., 13-18 August 2006, Belo Horizonte, Brazil.

[2] Bidinost F., Roldan D.L., Dodero A.M., Cano E.M., Taddeo H.R., Mueller J.P., Poli M.A., Quantitative trait loci related to Merino sheep quality, Proc. 8th World Congr. Genet. Appl. Livest. Prod., 13-18 August 2006, Belo Horizonte, Brazil.

[3] Cloete S.W.P., Greeff J.C., Lewer R., Direct and maternal genetic (co)variances for hogget live weight and fleece traits in Western Australian Merino sheep, Aust. J. Agric. Res. 53 (2002) 271-279.

[4] Falconer D.S., Mackay T.F.C., Introduction to quantitative genetics, Longman Group Ltd., England (1996).

[5] Gilmour A.R., Thompson R., Cullis B.R., ASREML, Biometrics Bulletin No. 3 (1998).

[6] Gootwine E., Zenou A., Bor A., Yossafi S., Rosov A., Pollott G.E., Introgression of the callipyge mutation into the Assaf fat tail breed, Options Méditerranéennes Série A, Séminaires Méditerranée 55 (2002) 125-131.

[7] Hatcher S., Atkins K., Swan A.A., Purvis I.W., Does translocating sheep between environments affect wool production and wool quality of Merino wethers? Aust. Assoc. Anim. Breed. Genet. 13 (1999) 106-109.

[8] Henry H.M., Dodds K.G., Wuliji T., Jenkins Z.A., Beattie A.E., Montgomery G.W., A genome screen for QTL for wool traits in a Merino $\times$ Romney backcross flock, Wool Tech. Sheep Breed. 46 (1998) 213-217.

[9] Parson Y.M, Cooper D.W., Piper L.R., Evidence of linkage between highglycine-tyrosine keratin gene loci and wool fibre diameter in a Merino half-sib family, Anim. Genet. 25 (1994) 105-108.

[10] Ponz R., Moreno C., Allain D., Elsen J.M., Lantier F., Lantier I., Brunel J.C., Perez-Enciso M., Assessment of genetic variation explained by markers for wool traits in sheep via a segment mapping approach, Mamm. Genome 12 (2001) 569-572.

[11] Purvis I.W., Franklin I.R., Major genes and QTL influencing wool production and quality: a review, Genet. Sel. Evol. 37 Suppl. 1 (2005) 97-101.

[12] Purvis I.W., Swan A.A., Can follicle density be used to enhance the rate of genetic improvement in Merino flocks? Aust. Assoc. Anim. Breed. Genet. 12 (1997) 512-515.

[13] Southey B.R., Thomas D.L., Gottfredson R.G., Zelinsky R.D., Ewe productivity of Booroola Merino-Rambouillet crossbred sheep during early stages of introgression of the Fec(B) allele into a Rambouillet population, Livest. Prod. Sci. 75 (2002) 33-44.

[14] van der Waaij E.H, van Arendonk J.A.M., Introgression of genes responsible for disease resistance in a cattle population selected for production: genetic and economic consequences, Anim. Sci. 70 (1999) 207-220.

[15] Visscher P.M., Haley C.S., On the efficiency of marker-assisted introgression, Anim. Sci. 68 (1999) 59-68.

[16] Visscher P., Pong-Wong R., Whittemore C., Haley C., Impact of biotechnology on (cross)breeding programmes in pigs, Livest. Prod. Sci. 65 (2000) 57-70. 